\title{
Universality of NMR Results in LISICON Systems and Other Solid Lithium Conductors
}

\author{
R. Bertermann* and W. Müller-Warmuth \\ Institut für Physikalische Chemie der Westfälischen Wilhelms-Universität, \\ Schlossplatz 4/7, D-48149 Münster, Germany
}

Z. Naturforsch. 53 a, 863-873 (1998); received August 22, 1998

\begin{abstract}
The temperature evolution of the ${ }^{7} \mathrm{Li}$ NMR spectra and relaxation rates in many investigated solid lithium conductors shows more or less the same behavior. These compounds are characterized by a disordered lithium sublattice with a surplus of cation sites in cavities and channels of the anionic network. At low temperature, the spectra consist of a central line and a distributed satellite base structure. Upon increasing temperature, both components narrow until a reduced constant width with a well resolved quadrupole structure is reached. The mean nuclear quadrupole coupling parameters reduce by either about 5 or by a factor of 15 in all the compounds. The spin-lattice relaxation rates $1 / T_{1}$ are asymmetric as a function of reciprocal temperature and of quadrupolar origin. The activation energy of the main process of ionic motion may best be obtained from the temperature dependence of the dipolar spin-spin-relaxation rate $1 / T_{2}$. The spectral densities of the relaxation dependences correspond to those for inhomogeneous motions; they may be described by modification of the BPP equation, a Cole-Davidson distribution or a Kohlrausch-Williams-Watts function. Within this study three LISICON systems, $\mathrm{Li}_{4-3 x} \mathrm{Ga}_{x} \mathrm{GeO}_{4}$, and two phosphates $\mathrm{Li}_{3} \mathrm{M}_{2}\left(\mathrm{PO}_{4}\right)_{3}(\mathrm{M}=\mathrm{Sc}$, In) were investigated or re-investigated which fit well into this scheme. Activation energies of $39-43 \mathrm{~kJ} / \mathrm{mol}$ $\left(\mathrm{Li}_{4-3 x} \mathrm{Ga}_{x} \mathrm{GeO}_{4}\right.$ with $\left.x=0.06,0.14,0.24\right), 53 \mathrm{~kJ} / \mathrm{mol}\left(\mathrm{Li}_{3} \mathrm{Sc}_{2}\left(\mathrm{PO}_{4}\right)_{3}\right)$ and $75 \mathrm{~kJ} / \mathrm{mol}\left(\mathrm{Li}_{3} \mathrm{In}_{2}\left(\mathrm{PO}_{4}\right)_{3}\right)$ were obtained.
\end{abstract}

\section{Introduction}

Lithium conducting materials have been given much attention in connection with the application of superionic conductors. NMR studies of the lithium dynamics in various systems with high ionic conductivity have shown many similarities independent of the chemical composition of the specific compound. All these materials possess channels and cavities and a disordered lithium sublattice with a surplus of potential cation sites in which the cations may move.

NMR, at least in principle, provides a wealth of information. Previous studies of the temperature and frequency dependence of ${ }^{7} \mathrm{Li}$ NMR spectra and spinlattice $\left(T_{1}\right)$ and spin-spin relaxation times $\left(T_{2}\right)$ of various thioborate conductors $[1-4]$ revealed that the same type of line narrowing process with a well resolved quadrupole structure (in crystalline compounds) at high temperatures and characteristical

\footnotetext{
* Present address, D-97074 Würzburg, Am Hubland, Institut für Anorganische Chemie.

Reprint requests to Prof. W. Müller-Warmuth; Fax: +49 251 8323441.
}

$1 / T_{1}$ and $1 / T_{2}$ dependences occur in all the materials. These features are similar to those already observed in other solid electrolytes [5], for instance in $\mathrm{Li}_{3} \mathrm{In}_{2}\left(\mathrm{PO}_{4}\right)_{3}[6,7]$ and $\mathrm{Li}_{3} \mathrm{Sc}_{2}\left(\mathrm{PO}_{4}\right)_{3}$ [8]. We wonder therefore whether there is a certain "universality" as far as the NMR results in combination with certain structural prerequisites are concerned.

Within the frame of the present study we looked into this question and studied further materials such as LISICON systems and lithium metal phosphates. LISICON is an acronym for lithium superionic conductor, but it means in the stricter sense solid solutions with $\gamma-\mathrm{Li}_{3} \mathrm{PO}_{4}$ structure formed by $\mathrm{Li}_{4} \mathrm{XO}_{4}(\mathrm{X}=$ $\mathrm{Si}, \mathrm{Ge}, \mathrm{Ti})$ with $\mathrm{Li}_{3} \mathrm{YO}_{3}(\mathrm{Y}=\mathrm{P}, \mathrm{As}, \mathrm{V})$ or $\mathrm{Li}_{2} \mathrm{MXO}_{4}$ $(\mathrm{M}=\mathrm{Zn}, \mathrm{Mg})$ or $\mathrm{Li}_{2} \mathrm{ZO}_{4}(\mathrm{Z}=\mathrm{S}, \mathrm{W})$ or $\mathrm{LiTO}_{4}(\mathrm{~T}=$ $\mathrm{Al}, \mathrm{Ga}$ ) [9]. Many compounds within these groups have been found to possess high ionic conductivity, especially solid solutions containing $\mathrm{Ga}$ in $\mathrm{Li}_{4} \mathrm{GeO}_{4}$ $[9,10]$. In this study we investigated $\mathrm{Li}_{4-3 x} \mathrm{Ga}_{x} \mathrm{GeO}_{4}$ with $x=0.06,0.14$ and 0.24 .

Furthermore, in view of the general question of the universality, we re-examined the phosphates $\mathrm{Li}_{3} \mathrm{Sc}_{2}\left(\mathrm{PO}_{4}\right)_{3}$ and $\mathrm{Li}_{3} \mathrm{In}_{2}\left(\mathrm{PO}_{4}\right)_{3}$. These compounds were already studied by Pronin, Vashman and 
Sigaryov [6 - 8], but some of these measurements had to be checked, in particular in view of the various phase transitions. More important, a substantial part of the interpretation is at variance with our findings.

\section{Experimental Details and Sample Structure}

Synthesis of the LISICON systems $\mathrm{Li}_{4-3 x} \mathrm{Ga}_{x}$ $\mathrm{GeO}_{4}$ was carried out similar to that described in [9], but the temperature program had to be changed. $\mathrm{Li}_{2} \mathrm{CO}_{3}, \mathrm{Ga}_{2} \mathrm{O}_{3}$ and $\mathrm{GeO}_{2}$ were purchased from Strem Chemicals with a chemical purity better than 99.99\%. Graphite crucibles in an atmosphere of argon were used. X-ray powder diffraction patterns of the final samples of $\mathrm{Li}_{4-3 x} \mathrm{Ga}_{x} \mathrm{GeO}_{4}$ with $x=0.06$, 0.14 and 0.24 were examined to control the quality of the products. A sample of lithium scandium phosphate, $\mathrm{Li}_{3} \mathrm{Sc}_{2}\left(\mathrm{PO}_{4}\right)_{3}$, was kindly provided by Sigaryov; more details as far as his synthesis, characterization and NMR results are concerned can be found in [8]. We verified the purity of the sample by $\mathrm{X}$-ray powder diffraction and realized by EPR and atomic spectroscopy the presence of copper, manganese and iron impurities of about 0.1 weight $\%$ each.

$\mathrm{Li}_{3} \mathrm{In}_{2}\left(\mathrm{PO}_{4}\right)_{3}$ was synthesized by a standard solid state reaction from $\mathrm{Li}_{2} \mathrm{CO}_{3}, \mathrm{In}_{2} \mathrm{O}_{3}$ and $\mathrm{NH}_{4} \mathrm{H}_{2} \mathrm{PO}_{4}$ (Strem Chemicals, purity better than $99.99 \%$ ), similar as described in [6] and [7]. In order to obtain the monoclinic modification it was necessary to anneal at $T>1290 \mathrm{~K}$ [11]. The product was again examined by X-ray powder diffraction, and the crystal structure data were found to agree with those of [11].

In addition, all the samples were investigated by differential scanning calorimetry (DSC) with a Netzsch DSC 200 apparatus. No phase transition and no special thermal effects were found for the $\mathrm{Li}_{4-3 x} \mathrm{Ga}_{x} \mathrm{GeO}_{4}$ compounds, in agreement with [9]. The DSC diagrams of the lithium phosphates (Fig. 1) show two maxima for $\mathrm{Li}_{3} \mathrm{Sc}_{2}\left(\mathrm{PO}_{4}\right)_{3}$, one weak maximum at $309 \mathrm{~K}$ and a phase transition at $536 \mathrm{~K}$. For $\mathrm{Li}_{3} \mathrm{In}_{2}\left(\mathrm{PO}_{4}\right)_{3}$ three possible phase transitions $(386 \mathrm{~K}$, $390 \mathrm{~K}, 415 \mathrm{~K}$ ) were observed.

The structure of the LISICON is derived from that of $\gamma-\mathrm{Li}_{3} \mathrm{PO}_{4}$ by the substitution mechanism $3 \mathrm{Li} \Leftrightarrow \mathrm{Ga}[9,10]$. The framework of $\mathrm{Li}_{4} \mathrm{GeO}_{4}$ is formed by $\mathrm{GeO}_{4}$ and $\mathrm{LiO}_{4}$ tetrahedra, and no disorder of lithium was observed. Substituted gallium occupies lithium sites, and the negative charge of the new pseudo-framework is compensated by "excess"
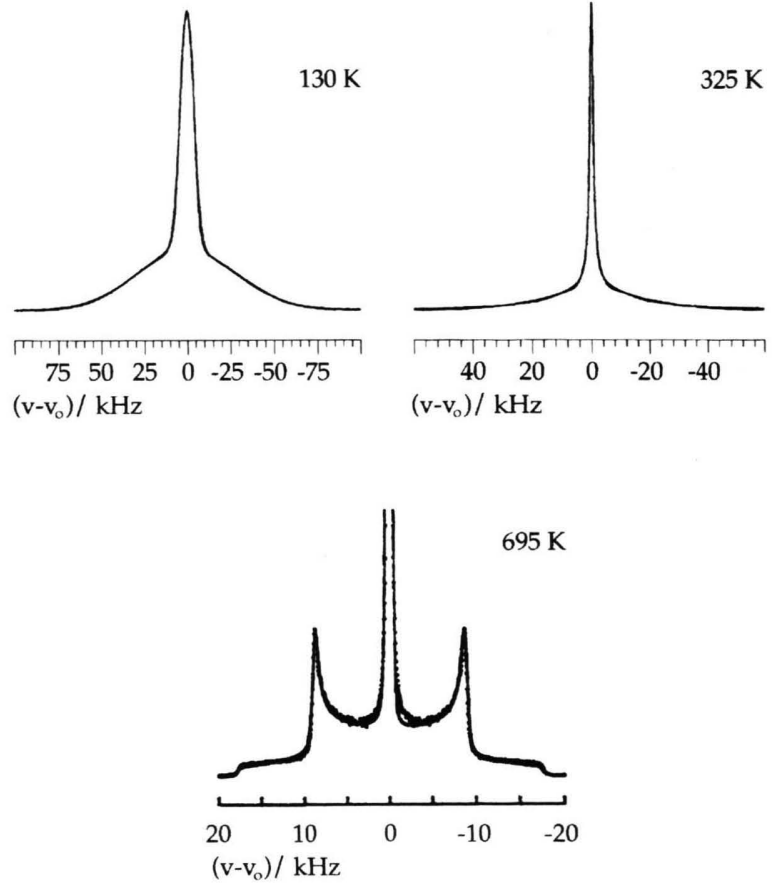

Fig. 1. ${ }^{7} \mathrm{Li}$ NMR spectra of $\mathrm{Li}_{3.28} \mathrm{Ga}_{0.24} \mathrm{GeO}_{4}$ at various temperatures. $\nu-\nu_{0}=0 \mathrm{kHz}$ corresponds to the resonance of a saturated $\mathrm{LiCl}$ solution as reference.

lithium cations in interstitial positions. These are weakly bound and may move in the channel system of the structure.

The room temperature phases of the lithium metal phosphates $\mathrm{Li}_{3} \mathrm{M}_{2}\left(\mathrm{PO}_{4}\right)_{3}(\mathrm{M}=\mathrm{Sc}, \mathrm{In})$ are isostructural and consist of $\mathrm{MO}_{6}$ octahedra and $\mathrm{PO}_{4}$ tetrahedra which are linked by sharing corner oxygen atoms [6 - 8, 12 - 14]. An infinite three-dimensional $\left[\mathrm{M}_{2} \mathrm{P}_{3} \mathrm{O}_{12}\right]^{3-}$ framework is formed which provides channel-like cavities. The difference between the various polymorphic modifications seems to be mainly caused by the difference in the $\mathrm{Li}^{+}$cation distribution and small changes in the atomic distances [12].

NMR studies were carried out at two frequencies, 116.64 $\mathrm{MHz}$ and $16.1 \mathrm{MHz}$, and at temperatures between $90 \mathrm{~K}$ and $700 \mathrm{~K}$. Methods and conditions are more or less the same as those in preceding papers [1-4].

\section{Experimental Results}

\subsection{LISICON Systems}

The ${ }^{7} \mathrm{Li}$ NMR spectra of $\mathrm{Li}_{4-3 x} \mathrm{Ga}_{x} \mathrm{GeO}_{4}$ with $x$ $=0.06,0.14,0.24$ look rather similar: Three charac- 


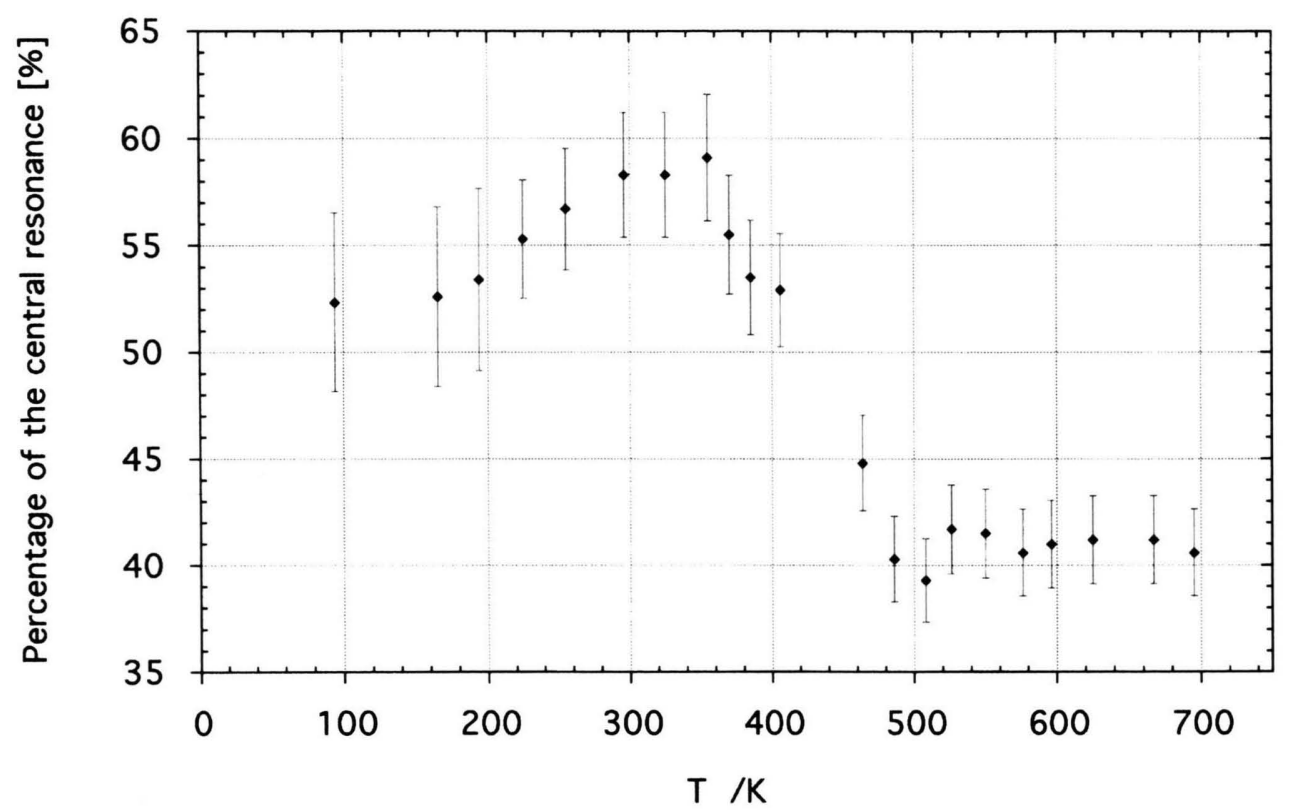

Fig. 2. Share of the peak-area of the central resonance to the total ${ }^{7} \mathrm{Li} \mathrm{NMR}$ spectrum of $\mathrm{Li}_{3.28} \mathrm{Ga}_{0.24} \mathrm{GeO}_{4}$ as a function of temperature.

teristic temperature ranges can be distinguished. At temperatures below about $245 \mathrm{~K}$, the spectra consist of an intense central resonance and a broad base. Above this temperature $T_{\mathrm{c}}$, which is only slightly dependent on composition, line narrowing occurs. Both the central transition and the broad base narrow continuously until about $350 \mathrm{~K}$. Above this temperature quadrupole structures arise; they become more and more pronounced, and above $500 \mathrm{~K}$ there is no further change or narrowing. Figure 1 shows the three characteristic limiting shapes of the spectra for one of the compounds.

The low temperature spectra can be simulated by two Gaussians with (total) widths $\Delta \nu_{\text {r.l. }}$. Upon increasing temperature both components become Lorentzian in shape. The activation energy of the line narrowing process can be estimated from the onset temperature $T_{\mathrm{c}}$ using the semiempirical formula [15]

$$
E_{\mathrm{A}}(\mathrm{W} . \mathrm{F} .) / \mathrm{kJ} \mathrm{mol}^{-1} \approx 0.156 T_{\mathrm{c}} / \mathrm{K} \text {. }
$$

The satellite structure at high temperatures can be simulated by an NMR spectrum with first order quadrupole interaction, a nuclear quadrupole coupling constant $\chi_{\mathrm{Q}}=e^{2} q Q / h$ (with $e Q$ and $e q$ being the nuclear quadrupole moment and the maximum component of the electric field gradient tensor in its
Table 1. Characteristic parameters of the ${ }^{7} \mathrm{Li}$ NMR spectra of the LISICON compounds as explained in the text.

\begin{tabular}{lccc}
\hline & $\mathrm{Li}_{3.82} \mathrm{Ga}_{0.06}-\mathrm{Li}_{3.58} \mathrm{Ga}_{0.14}-\mathrm{Li}_{3.28} \mathrm{Ga}_{0.24}$ \\
& $\mathrm{GeO}_{4}$ & $\mathrm{GeO}_{4}$ \\
\hline Rigid lattice range & $T<248 \mathrm{~K}$ & $T<245 \mathrm{~K}$ & $T<240 \mathrm{~K}$ \\
$\Delta \nu_{\text {r.l. }}$ (central)/kHz & 10.7 & 11.6 & 11.4 \\
$\Delta \nu_{\text {r.l. }}$ (base)/kHz & 69 & 80 & 81 \\
Line narrowing range & \multicolumn{3}{c}{$-245 \mathrm{~K} \leq T \leq 350 \mathrm{~K}-$} \\
$E_{\mathrm{A}}$ (W.F.)/kJ/mol & \multicolumn{3}{c}{$-38-$} \\
Quadrupole structure & \multicolumn{3}{c}{$-\mathrm{T}>350 \mathrm{~K}-$} \\
$\chi_{\mathrm{Q}} / \mathrm{kHz}$ & 32 & 34 & 35 \\
$\eta$ & 0.16 & 0.09 & 0 \\
\hline
\end{tabular}

principal axes system, as usual) and an asymmetry parameter $\eta$. Data for the LISICON compounds, collected from the observation of the ${ }^{7} \mathrm{Li}$ NMR spectra at various temperatures, are listed in Table 1.

In order to understand the evolution of the spectra with increasing temperature, we also measured the proportions of the area of the central resonance to the area of the broad unstructured (low temperature) or structured (high temperature) base of the peak. Figure 2 gives the percentage of the central line intensity at various temperatures. Both at low and high temperatures, the area ratio amounts to $40-50 \%$, as to be expected for a $I=3 / 2$ spectrum with satellites due to 


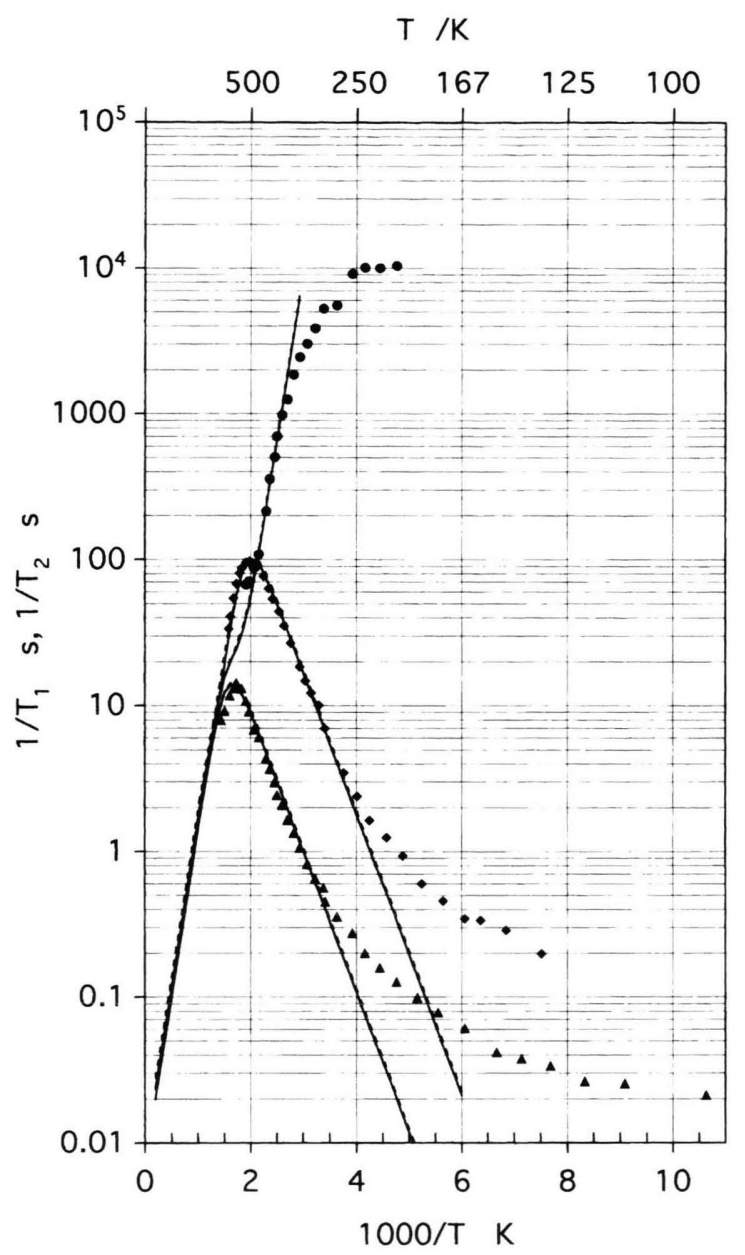

Fig. 3. Spin-lattice relaxation rates measured at $117 \mathrm{MHz}$ $(\boldsymbol{\Delta})$ and $16.1 \mathrm{MHz}(\bullet)$ and spin-spin-relaxation rates $(117 \mathrm{MHz}: \bullet)$ plotted versus reciprocal temperature for $\mathrm{Li}_{3.28} \mathrm{Ga}_{0.24} \mathrm{GeO}_{4}$. The lines are the result of the best fits by (2) and (3) with either the Cole-Davidson distribution (solid line) or the modified BPP equation (broken line) and the parameters given in Table 2. More details are discussed in the text. The very low temperature process is not considered.

first order quadrupole interaction. In the motional narrowing regime part of the satellite intensity flows into the central line, but as the quadrupole structure builts up at high temperatures, the satellite spectrum regains its full intensity. Since it is difficult to excite the broad quadrupole satellites completely, at low temperatures the error is larger and the central resonance is probably overvalued.

Figure 3 shows the spin-lattice and spin-spin relaxation rates at various temperatures and frequencies for
Table 2. Apparent activation energies extracted from the $T_{1}$ and $T_{2}$ relaxation measurements of $\mathrm{Li}_{4-3 x} \mathrm{Ga}_{x} \mathrm{GeO}_{4}$ (exp = experimental). The lower part collects the parameters used for the description of the relaxation rates by the modified BPP equation in Fig. 3 (mod. BPP) or by the Cole-Davidson distribution (C.D.).

\begin{tabular}{lccc}
\hline & $x=0.06$ & $x=0.14$ & $x=0.24$ \\
\hline Exp.: & & & \\
$E_{\mathrm{A}}^{\prime} / \mathrm{kJ} \mathrm{mol}^{-1}$ & $42 \pm 2$ & $45 \pm 6$ & $41 \pm 6$ \\
$E_{\mathrm{A}}^{\prime \prime} / \mathrm{kJ} \mathrm{mol}^{-1}$ & $20 \pm 2$ & $19 \pm 2$ & $19 \pm 2$ \\
$\mathrm{E}_{\mathrm{A}}\left(T_{2}\right) / \mathrm{kJ} \mathrm{mol}^{-1}$ & $39 \pm 3$ & $43 \pm 2$ & $43 \pm 2$ \\
$M o d . B P P:$ & & & \\
$E_{\mathrm{A}} / \mathrm{kJ} \mathrm{mol}{ }^{-1}$ & 42 & 45 & 44 \\
$C_{\mathrm{Q}} / \mathrm{s}^{-2}$ & $6.7 \cdot 10^{9}$ & $7.5 \cdot 10^{9}$ & $6.6 \cdot 10^{9}$ \\
$\alpha$ & 0.49 & 0.47 & 0.42 \\
$\tau_{\mathrm{co}} / \mathrm{s}$ & $1.0 \cdot 10^{-13}$ & $1.3 \cdot 10^{-13}$ & $2.5 \cdot 10^{-13}$ \\
$C . D .:$ & & & \\
$E_{\mathrm{A}} / \mathrm{kJ} \mathrm{mol}$ & & & \\
$C_{\mathrm{Q}} / \mathrm{s}^{-1}$ & 42 & 45 & 44 \\
$\alpha$ & $10 \cdot 10^{9}$ & $12 \cdot 10^{9}$ & $11 \cdot 10^{9}$ \\
$\tau_{\mathrm{co}} / \mathrm{s}$ & 0.49 & 0.50 & 0.42 \\
& $1.3 \cdot 10^{-13}$ & $1.5 \cdot 10^{-13}$ & $3.0 \cdot 10^{-13}$ \\
\hline
\end{tabular}

Table 3. Same as Table 1, but for the lithium metal phosphates.

\begin{tabular}{lcc}
\hline & $\mathrm{Li}_{3} \mathrm{Sc}_{2}\left(\mathrm{PO}_{4}\right)_{3}$ & $\mathrm{Li}_{3} \mathrm{In}_{2}\left(\mathrm{PO}_{4}\right)_{3}$ \\
\hline Rigid lattice range & $T<353 \mathrm{~K}$ & $T<330 \mathrm{~K}$ \\
$\Delta \nu_{\text {r.l. }}$ (central)/kHz & 5.8 & 6.3 \\
$\Delta \nu_{\text {r.l. }}$ (base $) / \mathrm{kHz}$ & 94 & 59 \\
Line narrowing range & $353 \mathrm{~K} \leq T \leq 428 \mathrm{~K}$ & $330 \mathrm{~K} \leq T \leq 375 \mathrm{~K}$ \\
$E_{\mathrm{A}}($ W.F. $) / \mathrm{kJ} / \mathrm{mol}$ & 55 & 52 \\
Quadrupole structure & $428 \mathrm{~K} \leq T \leq 536 \mathrm{~K}$ & $390 \mathrm{~K} \leq T \leq 413 \mathrm{~K}$ \\
$\chi_{\mathrm{Q}} / \mathrm{kHz}$ & 43 & 8.0 \\
$\eta$ & 0.23 & 0.5 \\
Quadrupole structure & $T>536 \mathrm{~K}$ & $T>413 \mathrm{~K}$ \\
$\chi_{\mathrm{Q}} / \mathrm{kHz}$ & 23 & 12.3 \\
$\eta$ & 0.43 & 0.41 \\
\hline
\end{tabular}

the LISICON compound with $x=0.24$. Corresponding data for the two other compounds are similar, but with slightly different characteristic features. At all temperatures, the establishment of the longitudinal magnetization and the decay of the transversal magnetization were exponential. The upper part of Table 2 collects some apparent activation energies that can be extracted from the temperature gradients of the relaxation rates: $E_{\mathrm{A}}^{\prime}$ from the high temperature part of $1 / T_{1}$ (only partially accessible), $E_{\mathrm{A}}^{\prime \prime}$ from the low temperature part of $1 / T_{1}$ (the constant contribution at very low temperatures was subtracted), and $E_{\mathrm{A}}\left(T_{2}\right)$ from $1 / T_{2}$. Interpretation of these data and the lines through the points will be discussed in Section 4 . 

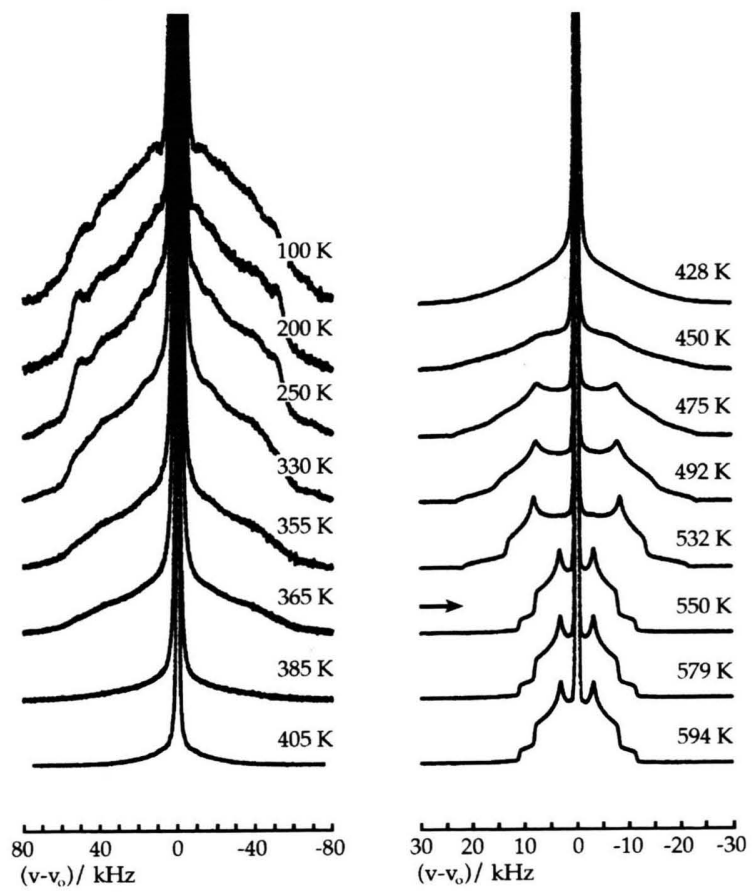

Fig. 4. Temperature dependence of the ${ }^{7} \mathrm{Li}$ NMR spectra of $\mathrm{Li}_{3} \mathrm{Sc}\left(\mathrm{PO}_{4}\right)_{3}$ observed at various temperatures. The arrow indicates a phase transition detected by DSC.
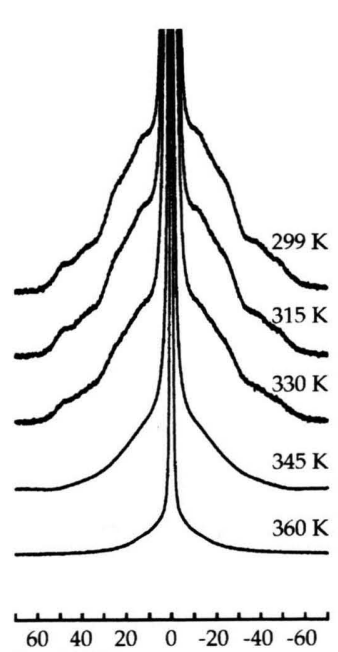
$\left(\mathrm{v}-\mathrm{v}_{\mathrm{o}}\right) / \mathrm{kHz}$
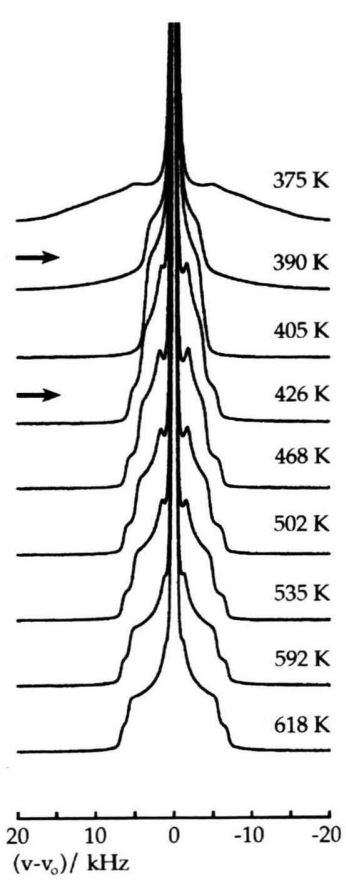

Fig. 5. Same as Fig. 4, but for $\mathrm{Li}_{3} \mathrm{In}_{2}\left(\mathrm{PO}_{4}\right)_{3}$.

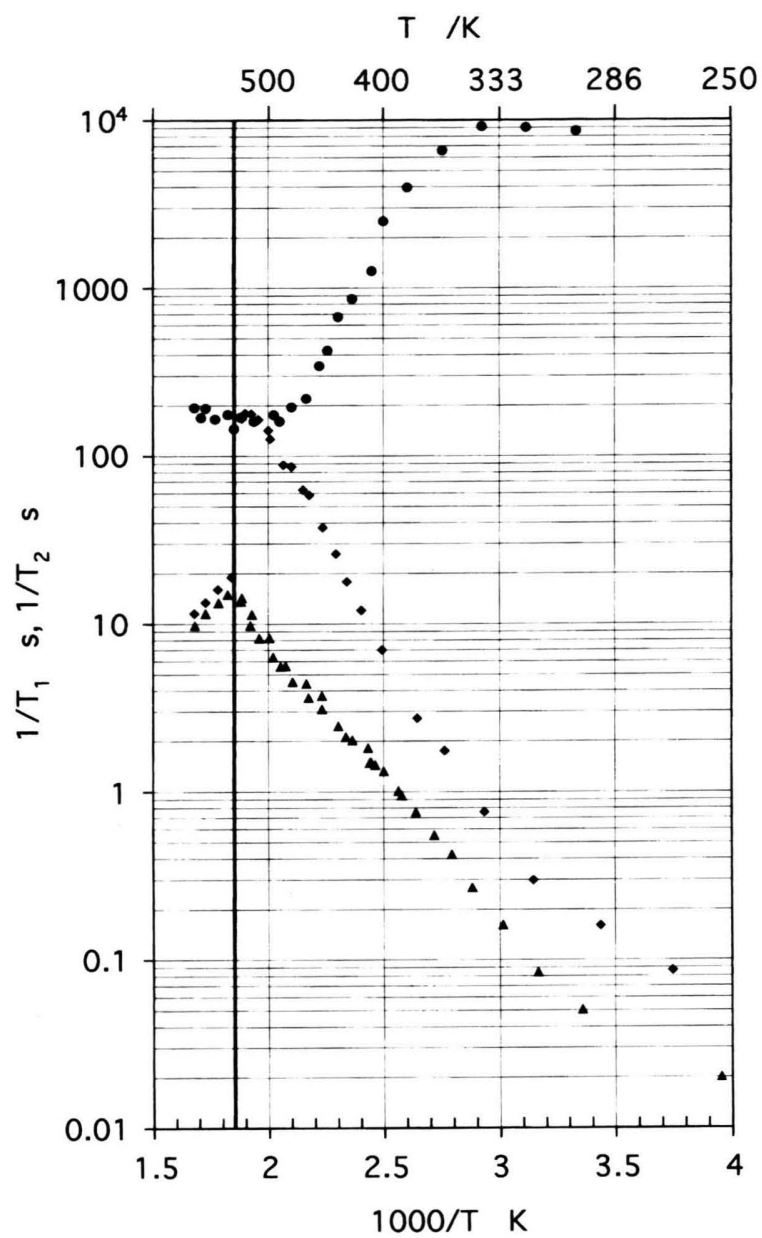

Fig. 6. ${ }^{7} \mathrm{Li}$ NMR spin-lattice and spin-spin relaxation rates for $\mathrm{Li}_{3} \mathrm{Sc}_{2}\left(\mathrm{PO}_{4}\right)_{3}$ plotted versus reciprocal temperature. The points refer to measurements at $117 \mathrm{MHz}\left(1 / T_{1}: \mathbf{\Lambda}, 1 / T_{2}: \bullet\right)$ and $16 \mathrm{MHz}\left(1 / T_{1}: \diamond\right)$. The phase transition at $536 \mathrm{~K}$ is indicated by a vertical line.

The ${ }^{7} \mathrm{Li}$ NMR spectra of the superionic phosphates $\mathrm{Li}_{3} \mathrm{Sc}_{2}\left(\mathrm{PO}_{4}\right)_{3}$ and $\mathrm{Li}_{3} \mathrm{In}_{2}\left(\mathrm{PO}_{4}\right)_{3}$ (Figs. 4 and 5) look in their temperature dependence similar to those of other lithium conductors, at least in principle. Special features are weakly structured satellites at very low temperatures (Figs. 4 and 5) and abrupt changes near the phase transition temperatures of $536 \mathrm{~K}$ in $\mathrm{Li}_{3} \mathrm{Sc}_{2}\left(\mathrm{PO}_{4}\right)_{3}$ (Fig. 4) and $386 \mathrm{~K} / 390 \mathrm{~K}$ and $413 \mathrm{~K}$ in $\mathrm{Li}_{3} \mathrm{In}_{2}\left(\mathrm{PO}_{4}\right)_{3}$ (Figure 5). Similar to Table 1 (for the LISICON systems), characteristic parameters for the various temperature ranges of both phosphates are listed in Table 3. At very low temperatures, only mean values of the broadening are given (as in Table 1) because the satellite structure is not at all well 


\section{$\mathrm{T} / \mathrm{K}$}

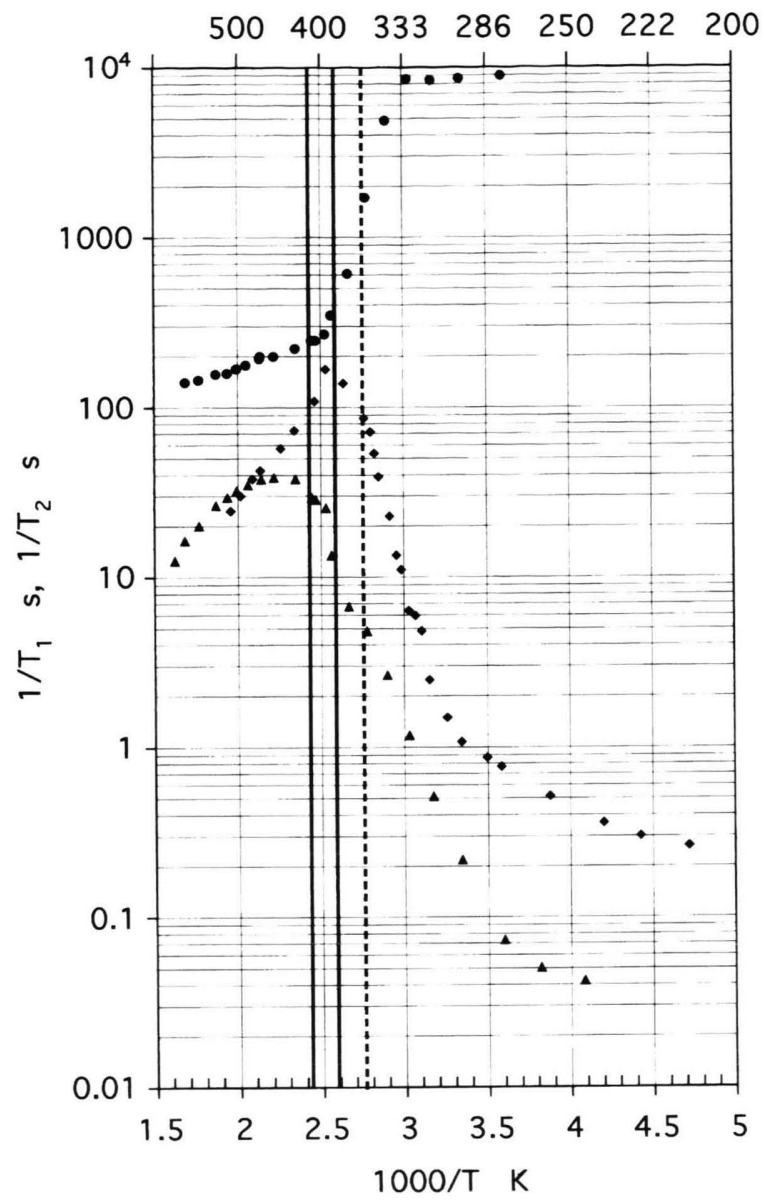

Fig. 7. Same as Fig. 6, but for $\mathrm{Li}_{3} \mathrm{In}_{2}\left(\mathrm{PO}_{4}\right)_{3}$ with two indicated phase transitions for the LT-modification (solid lines) and one for the HT modification (dotted).

resolved and seems to belong to the superposition of several electric field gradients. At high temperatures, different quadrupole structures were identified for the high temperature phases $(T>536 \mathrm{~K}$ and $T>413 \mathrm{~K}$, respectively) and the phases existing at intermediate temperatures.

We have also measured the spectral intensity at various temperatures for $\mathrm{Li}_{3} \mathrm{In}_{2}\left(\mathrm{PO}_{4}\right)_{3}$ (not shown). The percentage of the central resonance varies in a similar way as presented in Fig. 2 for the LISICON compound.

Figures 6 and 7 show the ${ }^{7} \mathrm{Li}$ NMR relaxation rates of the phosphates. Spin-spin and spin-lattice relaxation were both exponential. From the $1 / T_{2}$ dependence activation energies, $E_{\mathrm{A}}\left(T_{2}\right)=(53 \pm 3) \mathrm{kJ} / \mathrm{mol}$ for $\mathrm{Li}_{3} \mathrm{Sc}_{2}\left(\mathrm{PO}_{4}\right)_{3}$ and $E_{\mathrm{A}}\left(T_{2}\right)=(75 \pm 7) \mathrm{kJ} / \mathrm{mol}$ for
$\mathrm{Li}_{3} \mathrm{In}_{2}\left(\mathrm{PO}_{4}\right)_{3}$ were deduced. $1 / T_{1}$ shows in principle the same behaviour as in other lithium conductors, but a quantitative evaluation is not possible for various reasons. In Fig. 6, because of the phase transition at $536 \mathrm{~K}$ there is a jumplike dependence near the maximum of the $16 \mathrm{MHz}$ curve, and at $117 \mathrm{MHz}$ the maximum for a well defined phase is not accessible. Moreover, the convexity of the $16 \mathrm{MHz}$ curve between $400 \mathrm{~K}$ and $500 \mathrm{~K}$ reminds of what was observed and described in some detail for lithium conducting glasses containing iron impurities $[16,17]$. Quantitative information of the $1 / T_{1}$ curves of Fig. 7 suffers from the various phase transitions and modifications of the material, which cuts the temperature dependent curves in too many components. Maxima are not accessible.

\section{Discussion of Individual Results}

\section{1. $\mathrm{Li}_{4-3 x} \mathrm{Ga}_{x} \mathrm{GeO}_{4}$ Systems}

NMR results confirm the high cationic mobility of the $\mathrm{Li}^{+}$ions already concluded from ionic conductivity studies $[9,10]$. The activation energy of about 42 $\mathrm{kJ} / \mathrm{mol}$ obtained from the $T_{2}$ measurements (Table 2) agrees reasonably well with the $48 \mathrm{~kJ} / \mathrm{mol}$ derived from conductivity data. A satisfactory estimate of the activation energy is also available from the line narrowing process of the spectra (cf. Table 1: $38 \mathrm{~kJ} / \mathrm{mol}$ ) and from the high temperature gradient of the $1 / T_{1}$ curves (cf. Table 2: 41 - $45 \mathrm{~kJ} / \mathrm{mol}$ ).

There is also agreement with conductivity measurements as far as there are only very small differences at various concentrations $x$ of the gallium dopant (Tables 1 and 2). The activation energies are nearly the same within the error limits, the $1 / T_{1}$ maxima shift slighly to higher temperatures with increasing $x$ and the quadrupole couplings characteristic of the spectra increase a little bit. Most remarkable is the increasing symmetry of the electric field gradient at the lithium sites expressed in the descreasing asymmetry parameters $\eta$ of the high temperature spectra.

The Gaussian shape of the central line of the rigid lattice spectra (Fig. 1) indicates that the broadening results from dipole-dipole interactions. As for the materials studied in preceding papers [1 - 4], the broad base belongs to the satellites of first order quadrupole couplings which are smeared out. This is a consequence of a distribution of electric field gradients at the lithium sites. For $I=3 / 2$ nuclei the intensity of 
the central resonance should be $40 \%$ and that of the satellites $60 \%$. Figure 2 shows that this ratio is indeed derived from the experimental spectra at high temperatures, where the satellite structure is resolved and computer separation of the central and satellite intensities is easy. At low temperatures, agreement is satisfactory as well, considering that a part of the distributed satellite structure is hidden underneath the central resonance. We come back to the discussion of the intensity ratios in Section 5. Here we may conclude that line narrowing by anisotropic motion reduces the observable quadrupole width to about $1 / 6$ of its original value and the satellites become resolved.

The relaxation behaviour (Fig. 3) looks rather similar to that of lithium thioborate conductors [1 - 4]. The decay of $1 / T_{2}$ at increasing temperatures and the $1 / T_{1}$ curves with their maxima reveal one process of motion of the lithium ions which modulates both the magnetic dipole-dipole interactions and the electric quadrupole interactions "seen" by the ${ }^{7} \mathrm{Li}$ nuclei. Spin-spin relaxation time measurements carried out by $90^{\circ}-\tau-180^{\circ}-\tau$-echo sequences reflect the time dependence of the dipolar couplings; low temperature $1 / T_{2}$ values have the same order of magnitude as the rigid lattice central linewidth (cf. Table 1). At high temperatures, $1 / T_{2}$ may therefore become smaller than $1 / T_{1}$ which is of quadrupolar origin. The height of the $1 / T_{1}$ maxima is proportional to the square of the nuclear quadrupole coupling constant $\chi_{\mathrm{Q}}$.

Because of the asymmetry of the $1 / T_{1}$ curves in the presentation of Fig. 3, the low temperature branch does not mirror the real activation energy; this may better be deduced from $1 / T_{2}$. The high temperature branch is only partially accessible and not suited for the exact determination of the activation energy. At very low temperatures the motionally controlled relaxation process is superimposed by a relaxation mechanism which is little temperature dependent, but not relevant for the problem of the present study. Possible explanations, such as two-level tunnelling systems, localized motions in non rigid potentials and the influence of paramagnetic impurities, were discussed in previous papers $[2,3]$.

The anomalous frequency and temperature dependences of the relaxation rates are characteristic of relaxation in various inhomogeneous systems such as glasses, polymers and intercalation compounds. More details were already discussed in previous papers on unusual lithium dynamics in thioborate lithium conductors [2 - 4] and glasses [16, 18]. Further references
Table 4. Spectral density functions used for the description of the relaxation rates after (2) and (3): modified BPP equation ("mod. BPP"), Cole-Davidson distribution ("C.D.") and Kohlrausch-Williams-Watts function ("KWW"). References are given in the text.

\begin{tabular}{lrl}
\hline $\bmod$ BPP & $J(\omega)=$ & $\frac{\tau_{\mathrm{c}}}{1+\left(\omega \tau_{\mathrm{c}}\right)^{1+\alpha}}$ \\
C. D. & $J(\omega)=$ & $\int_{0}^{\infty} \frac{f(\tau)}{1+\omega^{2} \tau_{\mathrm{c}}^{2}} \mathrm{~d} \tau$ \\
& $f(\tau)=\frac{\sin \alpha \tau}{\pi}\left[\frac{\tau}{\tau_{\mathrm{c}}-\tau}\right]^{\alpha}, \tau \leq \tau_{\mathrm{c}}$ \\
& $f(\tau)=0, \tau>\tau_{\mathrm{c}}$ \\
KWW & $J(\omega)=\operatorname{Re} \int_{0}^{\infty} g(t) e^{-i \omega t} \mathrm{~d} t, g(t)=\exp \left\{-\left(t / \tau_{\mathrm{c}}\right)^{\alpha}\right\}$ \\
\hline
\end{tabular}

to this problem can also be found therein. The present results on $\mathrm{Li}_{4-3 x} \mathrm{Ga}_{x} \mathrm{GeO}_{4}$ systems provide an excellent example of such a behaviour, since large frequency and temperature ranges are accessible without phase transitions in between and covering one process of motion.

We described this process of motion observed at temperatures between about 250 and $700 \mathrm{~K}$ by the "weak collision" equations for the relaxation rates [19]

$$
\begin{aligned}
& \frac{1}{T_{1}}=C\left[J\left(\omega_{0} \tau_{\mathrm{c}}\right)+4 J\left(2 \omega_{0} \tau_{\mathrm{c}}\right)\right] \\
& \frac{1}{T_{2}}=C\left[\frac{3}{2} J(0)+\frac{5}{2} J\left(\omega_{0} \tau_{\mathrm{c}}\right)+J\left(2 \omega_{0} \tau_{\mathrm{c}}\right)\right],
\end{aligned}
$$

and inserted for the spectral density $J\left(\omega \tau_{\mathrm{c}}\right)$ one of the models presented in Table 4, the modified BPP equation [20,21], the Cole-Davidson distribution $[18,22]$ or the Kohlrausch-Williams-Watts function ("stretched exponential") [23]. Equation (3) is limited to shorter correlation times $\tau_{\mathrm{c}}$ where $\sqrt{M_{2}^{\text {r.l. }}} \tau_{\mathrm{c}}$ $\ll 1$ holds with $M_{2}^{\text {r.l. }}$ being the rigid lattice (dipolar) second moment of the (central) resonance curve. For $\sqrt{M_{2}^{\mathrm{r} .1} .} \tau_{\mathrm{c}}>1,1 / T_{2}$ reaches the plateau value which equals $\sqrt{2 / \pi} \sqrt{M_{2}^{\text {r.l. }}}$ for a Gaussian resonance curve. An Arrhenius type relationship was used for the temperature dependence of $\tau_{\mathrm{c}}$ with activation energy $E_{\mathrm{A}}$.

Figure 3 shows fits by the "mod. BPP" and "C. D." models, respectively, which appear to be equally good. The parameters are listed in Table 2 . In principle, the data can also be fitted by the KWW function (cf. Table 4), but less well, since the $1 / T_{1}-$ maxima appear broader than observed. The fits and the 
numerical values are rather similar for the three LISICON compounds (cf. Table 2). The deviations $\alpha$ from relaxation in homogeneous systems $(\alpha=1)$ amount to about 0.5 , similar to what was observed in other lithium ion conducting systems. The spin-lattice relaxation strength is determinde by quadrupole interactions $\left(C=C_{\mathrm{Q}}\right)$; it is proportional to $\chi_{\mathrm{Q}}^{2}$ and has the same order of magnitude as the quadrupolar part of the (broad) rigid lattice NMR-spectrum.

As a result, the NMR data describe a uniform process of diffusive motions in the LISICON systems which can be pursued and parameterized from low to high temperatures. Following the known structure of the materials $[9,10]$, the observed process depicts the random motion of lithium ions via the glut of interstitial cationic positions. NMR studies of zinc analogues of germanate LISICON systems $\mathrm{Li}_{16-2 x} \mathrm{Zn}_{x}\left(\mathrm{GeO}_{4}\right)_{4}$ [24] and of $\mathrm{Li}_{3+x} \mathrm{P}_{1-x} \mathrm{Si}_{x} \mathrm{O}_{4}$ $[25,26]$, though less detailed, showed in principle similar results. The apparent activation energies given in $[25,26]$ are of course too small since in these studies only the low temperature branches of $T_{1}$ were evaluated, and those do not reflect the real activation energies.

\section{2. $\mathrm{Li}_{3} \mathrm{Sc}_{2}\left(\mathrm{PO}_{4}\right)_{3}$}

The structural phase transition from monoclinic to orthorhombic at high temperatures was observed by DSC at $536 \mathrm{~K}$ in agreement with literature data. Relaxation time information is essentially available for the low temperature phase (cf. Fig. 6); in the evolution of the ${ }^{7} \mathrm{Li}$ NMR spectra (cf. Fig. 4) the phase transition leads to a further reduction of the quadrupole coupling by a factor of about two and an increase of the asymmetry parameter (cf. Table 3 ). Our experimental spectra and the essential features of $1 / T_{1}$ agree with those of [8], but the interpretation is different. Since only the low temperature slopes are accessible, $1 / T_{1}$ measurements do no provide much information. We have therefore measured $1 / T_{2}$ in addition, which gives an activation energy of $53 \mathrm{~kJ} / \mathrm{mol}$ in agreement with the estimate $E_{\mathrm{A}}$ (W.F.) from the line narrowing process of the spectra. But this value is smaller than that of $73 \mathrm{~kJ} / \mathrm{mol}$ obtained from conductivity measurements [14]. For all the other ionic conductors studied in this work there was agreement between the activation energies derived from conductivity and NMR $\left(T_{2}\right)$. A peculiarity of the rigid lattice spectra (Fig. 4) are weak structures which can be recognized in the smeared satellites. The electric field gradients at the lithium sites seem to be less distributed than in other lithium conductors, probably because of the crystal structure which provides only three sets of non-equivalent cationic positions [14]. The width of the central line has the same order of magnitude as $1 / T_{2}$ at very low temperatures; both are dominated by dipolar interactions. The line narrowing process and the development of well-resolved first order quadrupole structures at high temperatures are similar to those of the other materials. The intensity ratio of the central line and the satellites (resolved or not resolved) corresponds to that of the other compounds. The anomalous behaviour of the ${ }^{7} \mathrm{Li} \mathrm{NMR}$ intensity postulated in [8] could not be confirmed.

Inspection of the NMR measurements of $\mathrm{Li}_{3} \mathrm{Sc}_{2}$ $\left(\mathrm{PO}_{4}\right)_{3}$ shows that the process of lithium ion diffusion can be pursued from low to high temperatures as well, where, however, full information is only obtained on the monoclinic phase. $1 / T_{2}$ and its decrease upon increasing temperature as well as the central line and its narrowing are governed by dipolar $\mathrm{Li}-\mathrm{Li}$ interactions and their time dependences by ionic motions. $1 / T_{1}$ and the satellite spectra are dominated by interactions between the nuclear quadrupole moment of ${ }^{7} \mathrm{Li}$ and the electric field gradient at the lithium sites which become modulated by the motions. Since these motions are anisotropic both interactions are reduced by a certain factor ending up in a constant width of the spectrum at high temperature. We did not observe any evidence of $\mathrm{Li}-\mathrm{Li}$ ion pairs as proposed in [8].

\section{3. $\mathrm{Li}_{3} \mathrm{In}_{2}\left(\mathrm{PO}_{4}\right)_{3}$}

In principle, the results are quite similar to those of lithium scandium phosphate, but the interpretation suffers from the existence of various modifications and phase transitions. We prepared pure monoclinic material $\left(\mathrm{HT}-\mathrm{Li}_{3} \mathrm{In}_{2}\left(\mathrm{PO}_{4}\right)_{3}\right)$ but suspect that this modification is metastable, changing to LT- $\mathrm{Li}_{3} \mathrm{In}_{2}\left(\mathrm{PO}_{4}\right)_{3}$ with trigonal symmetry if the temperature programs are carried out during the measurements. Evidence for this was obtained from our DSC studies (temporally after the NMR measurements) resulting in the peaks at $386 \mathrm{~K}, 390 \mathrm{~K}$ and $415 \mathrm{~K}$ similar to what is indicated in [11] for LT- $-\mathrm{Li}_{3} \mathrm{In}_{2}\left(\mathrm{PO}_{4}\right)_{3}: 382 \mathrm{~K}$ and $403 \mathrm{~K}$ and in contrast to $363 \mathrm{~K}$ expected for $\mathrm{HT}-\mathrm{Li}_{3} \mathrm{In}_{2}\left(\mathrm{PO}_{4}\right)_{3}$.

As for $\mathrm{Li}_{3} \mathrm{Sc}_{2}\left(\mathrm{PO}_{4}\right)_{3}$ week quadrupole structures can be recognized in the rigid lattice satellite spectra (cf. Figure 5). At high temperatures, the phase transi- 
tions near $390 \mathrm{~K}$ and $415 \mathrm{~K}$ change the shape of the spectra. Quadrupole coupling parameters derived for temperatures between both transitions (cf. Table 3) agree within the error limits with those given in [7], interpreted as belonging to $\mathrm{HT}-\mathrm{Li}_{3} \mathrm{In}_{2}\left(\mathrm{PO}_{4}\right)_{3}$. Above $413 \mathrm{~K}$ we obtained values similar to those given in [6] for $\mathrm{LT}-\mathrm{Li}_{3} \mathrm{In}_{2}\left(\mathrm{PO}_{4}\right)_{3}$. The line narrowing process mirrors again the reduction of both dipolar and quadrupolar couplings by the anisotropic motions of the lithium ions by a constant factor.

The activation energy of $75 \mathrm{~kJ} / \mathrm{mol}$, deduced from the temperature dependence of $1 / T_{2}$ between about $300 \mathrm{~K}$ and $400 \mathrm{~K}$ (cf. Fig. 7), corresponds to the activation energy of the conductivity determined for LT- $\mathrm{Li}_{3} \mathrm{In}_{2}\left(\mathrm{PO}_{4}\right)_{3}$ at temperatures below $415 \mathrm{~K}[6,11]$. For HT- $-\mathrm{Li}_{3} \mathrm{In}_{2}\left(\mathrm{PO}_{4}\right)_{3}$, conductivity measurements [7] lead to little larger activation energies at temperatures below $360 \mathrm{~K}$ and to clearly smaller values above $370 \mathrm{~K}$. The $1 / T_{1}$ data are, in principle, similar to those of other lithium conductors, but in this case they provide little quantitative information because of the various phase transitions. Information from the low temperature part is limited in principle because of the asymmetry of such $1 / T_{1}$ curves and the unknown spectral density function of motion.

In our experiments there was no indication of anomalies in the intensity of the ${ }^{7} \mathrm{Li}$ NMR spectra or of the existence of $\mathrm{Li}^{+}-\mathrm{Li}^{+}$contact pairs as proposed in [6] and [7]. The distribution of intensities between central resonance and satellites (not shown) is similar to that presented for LISICON in Figure 2. Only in the high temperature limit, a complete separation is possible leading to the correct theoretical value for the central and satellite intensities.

\section{Discussion of the Universality of the Results}

This part of the discussion is based on the NMR results of the lithium ion conductors studied in this work and furthermore on the fast lithium thioborate conductors $\mathrm{Li}_{5} \mathrm{~B}_{7} \mathrm{~S}_{13}$ [3], $\mathrm{Li}_{9} \mathrm{~B}_{19} \mathrm{~S}_{33}$ [4], $\mathrm{Li}_{6+2 x}\left[\mathrm{~B}_{10} \mathrm{~S}_{18}\right] \mathrm{S}_{x}$ with $x \approx 2$ [2], and $\mathrm{Li}_{4-2 x} \mathrm{Sr}_{2+x} \mathrm{~B}_{10} \mathrm{~S}_{19}$ with $x \approx$ 0.27 [4]. The behaviour of lithium thioborate glasses is in some respect similar. The experimental results of the present paper and studies of other authors cited in the list of references confirm that the "universality" of the NMR behaviour is not limited to one class of materials only, but seems to be a general property of fast lithium conductors. Pre-conditions are the availability of suitable diffusion paths in cavities and channels and a disordered lithium sublattice with a surplus of cation sites.

The following features were observed in all the materials:

i) Rigid lattice ${ }^{7} \mathrm{Li}$ NMR spectra with first order quadrupole splitting. The satellites are smeared out by a distribution of electric field gradients at the lithium sites. In exceptional cases (this work) hints of structured satellites can be recognized. The amount of dipolar coupling can be estimated from the central line, that of quadrupolar coupling from the satellites;

ii) Line narrowing at increasing temperatures by a constant factor which is interpreted by the effect of anisotropic motions on both dipole-dipole (central line with Gaussian shape becoming Lorentzian) and quadrupole interactions (large base). The onset temperature provides a reasonable estimate of the activation energy, comparable with those obtained from relaxation;

iii) Well resolved NMR spectra with first order quadrupole interaction; width and quadrupole coupling constant are reduced by either a factor of about 5 or by 15 compared with the rigid lattice spectrum. In some cases both spectral ranges can be observed, in other cases the ranges are separated by a phase transition. In lithium conducting glasses items i) and ii) apply as well, but the spectra at high temperatures (iii) are not resolved $[18,27]$.

iv) Spin-lattice relaxation in both the laboratory and the rotating frame is governed by the quadrupole interactions which become randomly time-dependent by the motions. The relaxation strength is of the order of magnitude of the quadrupole contribution to the second moment of the rigid lattice resonance curve. Formal descriptions with spectral densities based on a modified BPP equation or a Cole-Davidson distribution of correlation times with distribution parameters between 0.4 and $0.5[1-4,16]$ are possible. Stretched exponentials can also be applied, but they do not fit quite so well.

v) Spin-spin relaxation is governed by dipoledipole interactions (as measured by proper techniques). The limiting value at low temperatures corresponds to the square root of the rigid lattice second moment of the central resonance, and the decrease upon increasing temperatures gives a better access to the activation energy than the model dependent evaluation of the spin-lattice relaxation.

Alternative interpretations of such data put up for discussion by Sigaryov et al. for the lithium metal 
phosphates $[6,8]$ - if correct - should be valid for all the lithium conductors because of the universality of the results. They are at variance with our findings. These authors considered whether the rigid lattice spectra may be a superposition of a narrow and a broad dipolar component belonging to dipole-dipole couplings of either more distant lithium nuclei or lithium pairs with a separation of only $100 \mathrm{pm}$ to $120 \mathrm{pm}$. In their model, metastable $\mathrm{Li}^{+}-\mathrm{Li}^{+}$ion contact pairs play an important role for the ion transport. Consequently, the $1 / T_{1}$ maximum should then be determined by dipole-dipole interactions as well and an alternative explanation is given for the high temperature spectra.

Experimental arguments against this concept are: The dependences of the intensity on temperature, for some materials hints of structured quadrupole satellites at low temperatures, observed quadrupole effects by ${ }^{6} \mathrm{Li} \mathrm{NMR}$, the $T_{1} / T_{2}$ ratio near maximum spinlattice relaxation, and the spin-spin relaxation measurements of the dipolar interactions. An argument against lithium clustering is also the isotropic chemical shift which remains the same (between $-2 \mathrm{ppm}$ and $+2 \mathrm{ppm}$ with reference to $\mathrm{LiCl}$ solutions) for all materials and temperatures. We carried out several measurements of the intensity ratio like those of Fig. 2, and within the error limits the satellite portion intensity was found to be 0.6 , as it should be for a quadrupole spectrum. Only in the range of line narrowing the apparent intensity ratio (Fig. 2) increases considerably because a part of the narrowed satellites is hidden beneath the central line.

The quadrupole coupling constants estimated from the width of the rigid lattice spectra are of the order of magnitude of $100-230 \mathrm{kHz}$ similar to those of lithium oxide (170 - $220 \mathrm{kHz})$ [18] and thioborate glasses $(100-130 \mathrm{kHz})$ [27]. If the atomic motions of the lithium ions were isotropic, the spectra would narrow continuously. The observed well-resolved spectra at high temperatures and threshold values with a constant reduction of widths and quadrupole coupling

[1] P. zum Hebel, B. Krebs, M. Grüne, and W. MüllerWarmuth, Solid State Ionics 43, 133 (1990).

[2] M. Grüne, W. Müller-Warmuth, P. zum Hebel, and B. Krebs, Solid State Ionics 66, 165 (1993).

[3] M. Grüne, W. Müller-Warmuth, P. zum Hebel, and B. Krebs, Solid State Ionics 78, 305 (1995).

[4] R. Bertermann, W. Müller-Warmuth, C. Jansen, F. Hiltmann, and B. Krebs, Solid State Ionics, in press. constants are a consequence of the anisotropy of the motion. The motionally narrowed spectra have more or less the same quadrupole coupling constants of either $30-40 \mathrm{kHz}$ or $9-13 \mathrm{kHz}$. As far as we know, there are no theoretical approaches available for such a situation where line narrowing by anisotropic translational motions results in particularly well-resolved spectra. Calculations of line shapes with structure at high temperature resulting from certain types of hindered rotational motion in the solid state are well known for a long time [28].

The processes of lithium motion are quite similar in all the ionic conductors. At low temperatures the ${ }^{7} \mathrm{Li}$ ions are randomly distributed among various sites available in excess. The anionic sublattice provides suitable sites and is responsible for the differences in both the electric field gradients and the barriers which hinder the diffusion. If the electric field gradient at the lithium sites is less distributed and the non-equivalency limited to few values, there are hints of structured spectra even at low temperatures. The mechanism of line narrowing and the type of the motional correlation function are also similar for various materials. Assignment to specific diffusion paths in the lattice is not possible, although in some cases we observed more than one process of motion. The activation energies are of course different for different materials; they are determined by the energy barriers provided by the anionic sublattice.

\section{Acknowledgements}

Support of this study by the Deutsche Forschungsgemeinschaft (DFG) is gratefully acknowledged. The authors would like to thank Dr. S.E. Sigaryov, Moscov, for valuable discussions at the beginning of our work and for putting some of his samples at our disposal. We are grateful to the "Anorganisch-Chemisches Institut" (Prof. B. Krebs and coworkers) of the Münster University for the assistance in sample preparation and characterization.

[5] Review: D. Brinkmann, Prog. NMR Spectrosc. 24, 527 (1992).

[6] I. S. Pronin, S. E. Sigaryov, and A. A. Vashman, Solid State Ionics 38, 9 (1990).

[7] I. S. Pronin, A. A. Vashman, and S. E. Sigaryov, Phys. Rev. B 48, 16463 (1993).

[8] A. A. Vashman, I. S. Pronin, and S. E. Sigaryov, Solid State Ionics 58, 201 (1992). 
R. Bertermann and W. Müller-Warmuth - Universality of NMR Results in LISICON Systems

[9] A. Robertson and A. R. West, Solid State Ionics 58, 351 (1992), and references therein.

[10] A. K. Ivanov-Shits and S. E. Sigaryov, Solid State Ionics 27, 89 (1988).

[11] Y. K. Naganovsky and S. E. Sigaryov, Solid State Ionics 50, 1 (1992).

[12] S. E. Sigaryov, E. A. Genkina, and B. A. Maximov, Solid State Ionics 37, 261 (1990).

[13] D. Tran Qui and S. Hamdoune, Acta Cryst. C 43, 397 (1987).

[14] A. B. Bykov, A. P. Chirkin, L. N. Demyanets, S. N. Doronin, E. A. Genkina, A. K. Ivanov-Shits, I. P. Kondratyuk, B. A. Maksimov, O. K. Mel'nikov, L. N. Muradyan, V. I. Simonov, and V. A. Timofeeva, Solid State Ionics 38, 31 (1990).

[15] J. S. Waugh and E. I. Fedin, Sov. Phys. Solid State 4, 1633 (1963).

[16] M. Grüne and W. Müller-Warmuth, Ber. Bunsenges. Physik. Chem. 95, 1068 (1991).

[17] M. Grüne and W. Müller-Warmuth, Solid State Nuclear Magnetic-Resonance 5, 145 (1995).
[18] E. Göbel, W. Müller-Warmuth, H. Olyschläger, and H. Dutz, J. Magn. Reson. 36, 371 (1979).

[19] A. Abragam, The Principles of Nuclear Magnetism, Oxford Univ. Press (Clarendon), London 1961.

[20] J. L. Bjorkstam and M. Villa, Phys. Rev. B 22, 5025 (1980).

[21] Cl. Ritter, W. Müller-Warmuth, and R. Schöllhorn, J. Chem. Phys. 83, 6130 (1985).

[22] D. W. Davidson and R. H. Cole, J. Chem. Phys. 15, 1417 (1950).

[23] G. Williams and D. C. Watts, Trans. Faraday Soc. 66, 80 (1970).

[24] M. Bose, A. Basu, and D. Torgenson, Solid State Ionics 18/19, 539 (1986).

[25] T. Asai and S. Kawai, Solid State Ionics 7, 43 (1982).

[26] M. Smaihi, D. Petit, F. Gourbilleau, F. Chaput, and J. P. Boilot, Solid State Ionics 48, 213 (1991).

[27] M. Grüne, H. Meierkord, W. Müller-Warmuth, P. zum Hebel, B. Krebs and M. Wulff, Ber. Bunsenges. Physik. Chem. 93, 1313 (1989).

[28] H. S. Gutowsky and G. E. Pake, J. Chem. Phys. 18, 162 (1950). 